\title{
Modeling of the Lyman $\gamma$ satellites in FUSE spectra of DA white dwarfs $\star$
}

\author{
G. Hébrard ${ }^{1}$, N. F. Allard ${ }^{1,2}$, J. F. Kielkopf ${ }^{3}$, P. Chayer $^{4}$, J. Dupuis ${ }^{4}$, J. W. Kruk ${ }^{4}$, and I. Hubeny ${ }^{5}$ \\ 1 Institut d'Astrophysique de Paris, CNRS, 98bis boulevard Arago, 75014 Paris, France \\ 2 Observatoire de Paris-Meudon, LERMA, 92195 Meudon Principal Cedex, France \\ 3 Department of Physics, University of Louisville, Louisville, KY 40292, USA \\ ${ }^{4}$ Department of Physics and Astronomy, Johns Hopkins University, Baltimore, MD 21218, USA \\ 5 NOAO, 950 North Cherry Avenue, Tucson, AZ 85726, USA
}

Received 1 April 2003 / Accepted 7 May 2003

\begin{abstract}
We present new theoretical calculations of the line profile of Lyman $\gamma$ that include transitions in which a photon is absorbed by a neutral hydrogen atom while it interacts with a proton. Models show that two absorption features located near $992 \AA$ and $996 \AA$ are due to $\mathrm{H}-\mathrm{H}^{+}$collisions. These quasi-molecular satellites are similar to those that were identified in the wings of Lyman $\alpha$ and Lyman $\beta$ lines of hydrogen-rich white dwarfs. We compute synthetic spectra that take account of these new theoretical profiles and compare them to the spectra of four DA white dwarfs that were observed with FUSE. The models predict the absorption features that are observed in the wing of Lyman $\gamma$ near $995 \AA$, and confirm that these features are quasi-molecular satellites.
\end{abstract}

Key words. line: profiles - radiation mechanisms: general - stars: atmospheres - white dwarfs - ultraviolet: stars

\section{Introduction}

Quasi-molecular satellites are absorption features due to transitions that take place during close collisions of a radiating hydrogen atom with a perturbing atom or proton. These spectral features, which are present in the red wings of the Lyman series lines, provide an important source of opacity in the atmospheres of hydrogen-rich white dwarfs.

The IUE spectra of the DA white dwarf 40 Eri B were the first ones which revealed a strong, unexpected absorption feature near $1400 \AA$ (Greenstein 1980). Absorption features near $1600 \AA$ were thereafter detected in spectra of cooler DA white dwarfs (Holm et al. 1985). These features remained unexplained until Koester et al. (1985) and Nelan \& Wegner (1985) simultaneously identified them as quasi-molecular satellites of the Lyman $\alpha$ line. The $\mathrm{H}_{2}$ and $\mathrm{H}_{2}^{+}$satellites at $1600 \AA$ and $1400 \AA$ were thereafter observed in other DA white dwarfs, as well as in laboratory plasmas (Kielkopf \& Allard 1995). The $\mathrm{H}_{2}^{+}$satellite absorption features at $1058 \AA$ and $1076 \AA$ were first identified in the spectrum of the DA white dwarf Wolf 1346, as observed with HUT (Koester et al. 1996). ORFEUS (Koester et al. 1998) and FUSE (Wolff et al. 2001; Hébrard et al. 2002b) observations allow these Lyman $\beta$ satellites to be observed in other targets.

Send offprint requests to: G. Hébrard, e-mail: hebrard@iap. fr

* This paper is dedicated in memory of J. L. Greenstein, discoverer of the quasi-molecular lines in white dwarfs, who passed away on October 21, 2002.
New calculations for the absorption profiles of Lyman $\gamma$ line of atomic hydrogen perturbed by protons have been used to predict synthetic spectra of hot hydrogen-rich white dwarfs and allow the identification of a strong feature near $995 \AA$ A. This feature was present in the HUT spectrum of Wolf 1346 (Koester et al. 1996) but it is only in Koester et al. (1998) that a similar feature present in ORFEUS spectra of WD 1031-114 and WD $0644+375$ was suspected to be a new satellite in the wing of Lyman $\gamma$. It was also distinctly detected in FUSE spectra of CD $-38^{\circ} 10980$ (Wolff et al. 2001) and of Sirius B (Holberg 2002).

These last two recent observations provided motivation to investigate the theory of the Lyman $\gamma$ line profile using accurate theoretical molecular potentials, instead of using the simple Stark broadening approximation. Our new calculations of Lyman $\gamma$ satellites are summarized in Sect. 2. We present in Sect. 3 a comparison between synthetic spectra including Lyman $\gamma$ satellites and the FUSE spectra of four DA white dwarfs. The results are discussed in Sect. 4.

\section{Theoretical line profiles}

The theoretical approach is based on the theory of pressure broadening due to Baranger (1958), developed in an "adiabatic representation" that does not exclude degeneracy of atomic levels. A detailed description of our semi-classical approach as applied to the shape of the Lyman lines has been given by Allard et al. (1994) and Allard et al. (1999). 
Table 1. Targets.

\begin{tabular}{lcccccccc}
\hline \hline Target & Observation & Obs. date & Mode & Slit & Exp. (ks) & CalFUSE $^{\mathrm{a}}$ & $T_{\text {eff }}$ & $\log g$ \\
\hline Wolf 1346 & B1190501 & Jul. 2001 & TTAG & LWRS & 5.8 & 1.8 .7 & 20000 & 8.0 \\
\hline EG 102 & B1190301 & May 2001 & TTAG & LWRS & 10.7 & 1.8 .7 & 20435 & 7.9 \\
& S6010401 & Jan. 2002 & TTAG & LWRS & 17.4 & 1.8 .7 & & \\
\hline BPM 6502 & Z9104501 & Jun. 2002 & TTAG & LWRS & 32.0 & 1.8 .7 & 21380 & 7.9 \\
\hline CD -38 10980 & Q1100101 & Jul. 2000 & HIST & MDRS & 4.8 & 2.2 .1 & 23000 & 7.9 \\
\hline
\end{tabular}

${ }^{a}$ Version of the pipeline used for spectral extraction.

The Lyman $\gamma$ profile and its satellites are calculated for physical conditions encountered in the atmospheres of white dwarfs. Because the density of the hydrogen atoms is low $\left(10^{15}\right.$ to $10^{17} \mathrm{~cm}^{-3}$ ), we computed the line profile by using the low density approximation as described by Allard et al. (1994). This approximation uses the expansion of the autocorrelation function in powers of density. We also used accurate theoretical potentials describing the binary interactions of one hydrogen atom with a ionized hydrogen atom. The $\mathrm{H}_{2}^{+}$molecular potentials are available for the $\mathrm{H}(n \leq 3)$ states (Madsen \& Peek 1971). We computed all those related to $n=4$ using a code kindly provided by J. Peek.

Our theoretical approach allows the variation of the radiative dipole moment during a collision. But through lack of appropriate data for Lyman $\gamma$, we assumed that the dipole moment for the transitions is constant. Such approximation has been applied to the calculations of the quasi-molecular satellites observed in the IUE and HST spectra of white dwarfs (see, e.g., Koester \& Allard 1993; Koester et al. 1994; Bergeron et al. 1995). In the last section we discuss the possible effects of a variable dipole moment on the line profile calculations.

An $\mathrm{H}_{2}^{+}$correlation diagram was constructed for Lyman $\gamma$ (Allard et al. 2003), to which 14 transitions contribute. Two of these transitions, $1 \mathrm{~s} \sigma_{g} \rightarrow 6 \mathrm{~h} \pi_{u}$ and $2 \mathrm{p} \sigma_{u} \rightarrow 7 \mathrm{i} \sigma_{g}$, yield satellites in the red portion of Lyman $\gamma$ at $992 \AA$ and $996 \AA$. Figure 1 shows the profiles of both individual satellites and their effect on the total profile of Lyman $\gamma$. We obtain a blend of the line satellites with a maximum near $992 \AA$. The profile gives a shape similar to the $995 \AA$ feature observed in white dwarf spectra.

\section{Astronomical applications to synthetic spectra for DA white dwarfs}

Atmosphere models have been calculated using the programs TLUSTY (Hubeny 1988; Hubeny \& Lanz 1992, 1995). We considered LTE model atmospheres with pure hydrogen composition that explicitly include the Lyman $\alpha$ and Lyman $\beta$ quasi-molecular opacities. The resulting spectra were computed by using the spectral synthesis code SYNSPEC that incorporates the quasi-molecular satellites of Lyman $\alpha$, Lyman $\beta$, and Lyman $\gamma$. Models predict that the Lyman $\gamma$ satellites are visible roughly for effective temperatures between $15000 \mathrm{~K}$ and $30000 \mathrm{~K}$. We verified that non-LTE effects are unimportant in the range of effective temperatures considered in this study.

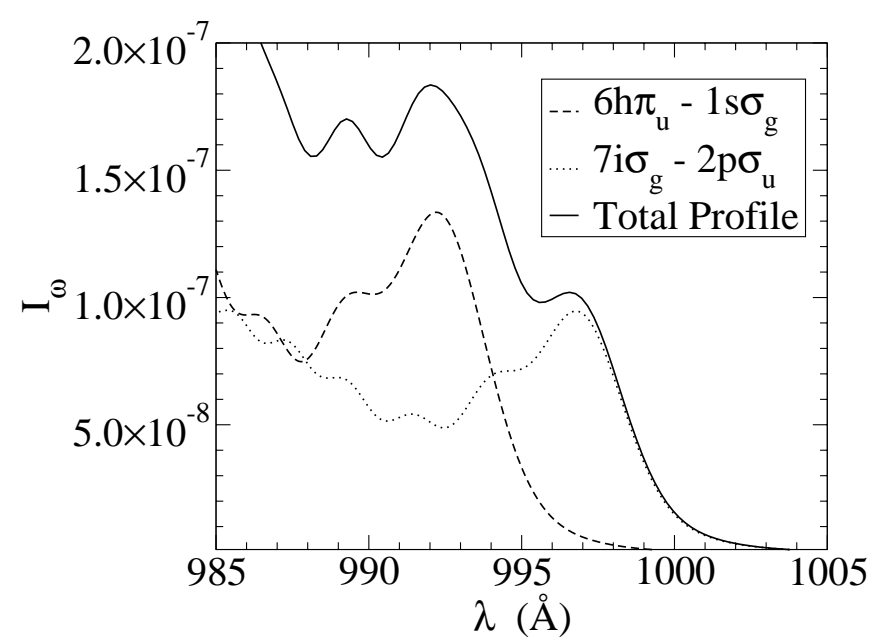

Fig. 1. The $995 \AA$-region Lyman $\gamma$ wing. The individual contribution of the two transitions that give rise to the two main satellites are overplotted. $I_{\omega}$ is the normalized line profile proportional to the absorption coefficient as described by Allard et al. (1999).

We used observations of four DA white dwarfs performed with FUSE (Moos et al. 2000). The spectra were obtained between 2000 and 2002 and were retrieved from the FUSE public database (see Table 1). The one-dimensional spectra were extracted from the two-dimensional detector images and calibrated using the CalFUSE pipeline. When possible, we did not use exposures with strong airglow emission. We also excluded regions of the spectra near the edges of the detector segments, which provide poor quality data. The eight FUSE detector segments of the different exposures were co-added together and projected on a $0.16 \AA$-pixel base, so pixels about 25 times larger than the original FUSE detector pixels. This degradation of the FUSE spectral resolution (typically $\lambda / \Delta \lambda \simeq 15000$ for this kind of target with the slit LWRS or MDRS; see Hébrard et al. 2002a or Wood et al. 2002) has no effect on the shapes of the large stellar features which we study and allows the signalto-noise ratio to be increased.

In Fig. 2 we compare the FUSE spectra with the models that we obtained for these targets. The effective temperatures are within the intermediate temperature range for the visibility of both the Lyman $\beta$ and Lyman $\gamma$ satellites. The agreement indicates, by the coincidence of position and approximate shape, that the large feature near $995 \AA$ is indeed a satellite of Lyman $\gamma$ due to the blend of two close features at $992 \AA$ and $996 \AA$. 

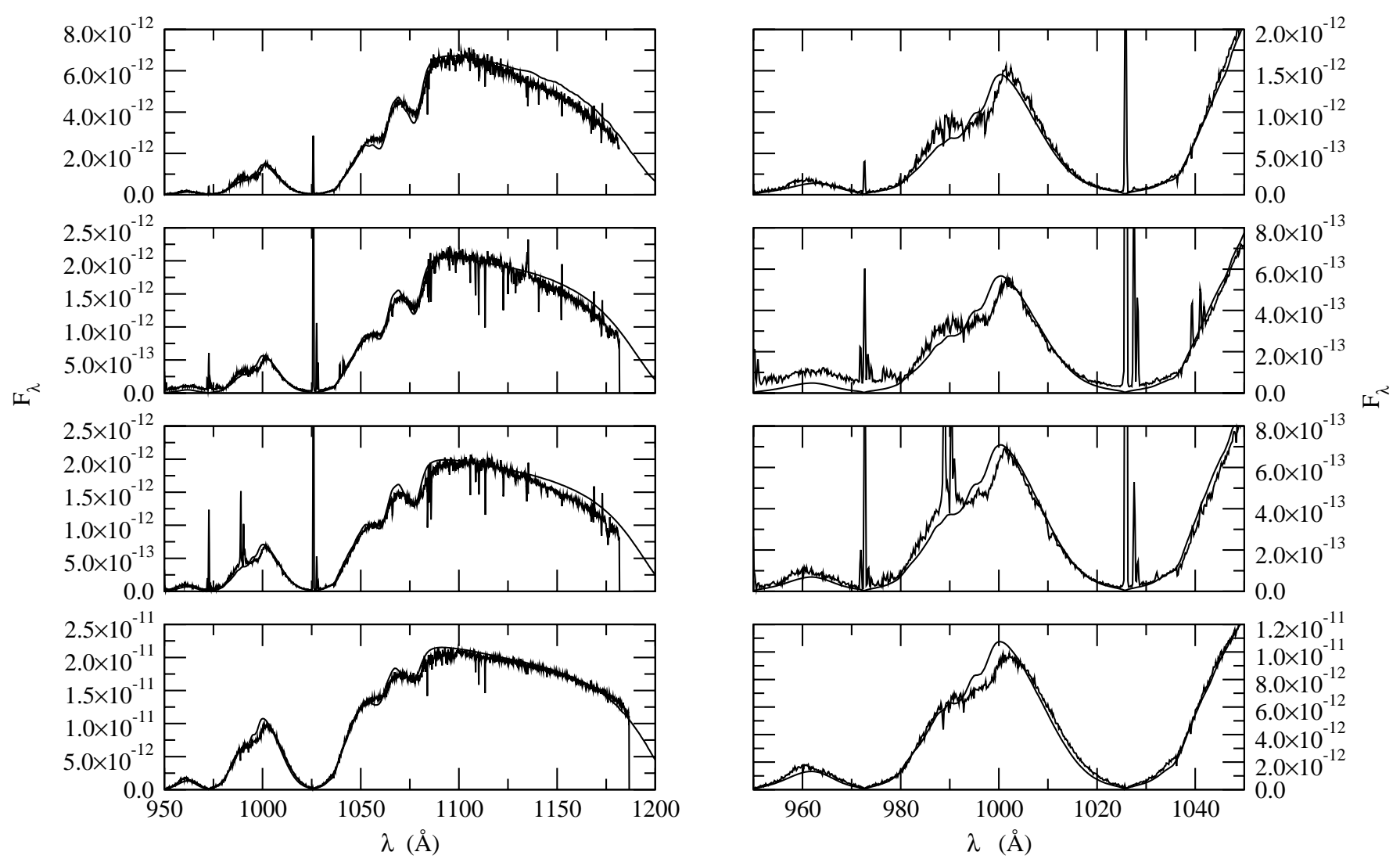

Fig. 2. A comparison of the FUSE spectra of the white dwarfs and the theoretical models. Top to bottom: Wolf 1346, EG 102, BPM 6502, and $\mathrm{CD}-38^{\circ} 10980$. The flux is in erg $/ \mathrm{cm}^{2} / \mathrm{s} / \AA$. The Lyman $\beta$ satellites are at $1058 \AA$ and $1076 \AA$; the Lyman $\gamma$ satellites (enlarged in right panel) are at $992 \AA$ and $996 \AA$. The narrow emission features are due to terrestrial airglow.

The first target plotted in Fig. 2 is Wolf 1346. The comparison for the FUSE spectrum shows that the far UV is well fitted with a synthetic spectrum computed with our new profile calculations for $\log g=8.0$ and $T_{\text {eff }}=20000 \mathrm{~K}$. These atmospheric parameters are close to those determined from optical spectra $(7.9 ; 20000)$ and adopted by Koester et al. (1996) to fit the HUT spectrum of that white dwarf. The Lyman $\gamma$ satellite, already observed in the HUT spectrum, is well-reproduced by our synthetic spectrum.

The next two objects, EG 102 and BPM 6502, are slightly hotter white dwarfs, with smaller gravities. We used the effective temperatures and gravities determined from optical spectra (Finley et al. 1997; Bragaglia et al. 1995). The predicted spectra for EG 102, with $T_{\text {eff }}=20435 \mathrm{~K}$ and $\log g=7.87$, and for BPM 6502, with $T_{\text {eff }}=21380 \mathrm{~K}$ and $\log g=7.86$, fit quite well the FUSE spectra. In the case of BPM 6502, all the FUSE exposures present strong airglow emissions, so it was not possible to remove them. These two stars are not normal DA white dwarfs: EG 102 presents metallic lines (Holberg et al. 1997) and BPM 6502 is in a post common-envelope binary system (Kawka et al. 2000, 2002). These particularities seem however to have no effects on the quality of the fits.

The hottest object we present is CD $-38^{\circ} 10980$. The FUSE observation that we used is the same as the one presented by Wolff et al. (2001), but our data reduction was performed with a more recent version of CalFUSE. Our best fit was obtained using $T_{\text {eff }}=23000 \mathrm{~K}$ and $\log g=7.9$. The values of the atmospheric parameters are slightly smaller than the ones determined from optical spectra (Vauclair et al. 1997) to get a better agreement with the Lyman $\gamma$ satellite.

$\mathrm{CD}-38^{\circ} 10980$ is near the upper limit of the expected visibility range of the Lyman $\beta$ satellites. Nevertheless, Lyman $\beta$ and Lyman $\gamma$ quasi-molecular satellites have been observed in the FUSE spectrum of the ultra-massive white dwarf PG $1658+441\left(T_{\text {eff }}=30500 \mathrm{~K}, \log g=9.36\right)$ as reported by Dupuis et al. (2001). We speculate that the presence of quasimolecular satellites in this star is related to its high surface gravity and we will investigate the question in more details in Dupuis et al. (2003). It is interesting to note that Sirius B $\left(T_{\text {eff }}=24790 \mathrm{~K}, \log g=8.57\right.$; Holberg et al. 1998), which also has a relatively high surface gravity, shows the quasi-molecular satellites (Holberg 2002).

Finally, the four spectra show a kink in the profile around $1037 \AA$ that is well reproduced by our models (Fig. 2). This feature is probably the first identification of the $\mathrm{H}_{2}^{+}$satellite of Lyman $\beta$, as predicted in Fig. 7 of Allard et al. (1998a)

\section{Conclusion}

The good agreement between the FUSE spectra and our calculations allowed the line satellites near $995 \AA$ to be identified, although there are still some systematic discrepancies between the predicted and observed profiles. The shape of the profile in the region of the satellites is sensitive to the relative strength 


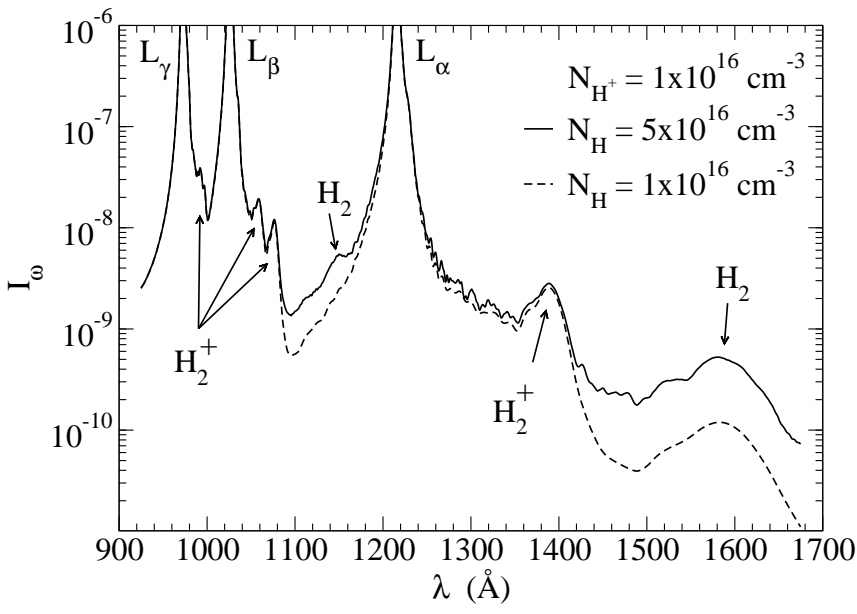

Fig. 3. Total profile of Lyman $\alpha$, Lyman $\beta$, and Lyman $\gamma$ perturbed by neutral hydrogen and protons. Two different neutral densities $(1 \times$ $10^{16} \mathrm{~cm}^{-3}$ and $5 \times 10^{16} \mathrm{~cm}^{-3}$ ) are compared for a fixed ion density $\left(1 \times 10^{16} \mathrm{~cm}^{-3}\right)$, and $T=25000 \mathrm{~K}$. Lyman $\gamma$ profile is only perturbed by protons.

of these two main features. It is therefore important to get an accurate quantitative determination of the satellite amplitudes. Accurate theoretical molecular potentials have to be used to describe the interaction between the radiator and the perturber as we did in the work described here. Another important factor, not yet included, is the variation of the dipole moment during the collision. Allard et al. (1998a,b, 1999) have shown that the strengths of line satellites are dependent on values of the electric-dipole moments at the internuclear separation responsible for the satellites. Large enhancements in the amplitudes of the satellites may occur whenever the dipole moment increases through the region of internuclear distance where the satellites are formed. The theoretical shape in this region of the profile is then dependent on the dipole moment of the transition. Some of the differences between the observed and the synthetic spectra may be due to our constant dipole moment approximation. That will be improved in future calculations.

As a summary we present in Fig. 3 the sum of the profiles of Lyman $\alpha$, Lyman $\beta$, and Lyman $\gamma$ perturbed by collisions with neutral hydrogen and protons for two different densities of neutral hydrogen. We can note that beside the quasi-molecular lines already detected, there is another predicted $\mathrm{H}_{2}$ satellite at $1150 \AA$ (Allard et al. 2000), in the red wing of Lyman $\beta$, which should be observed in cool DA white dwarfs in the range of effective temperatures (11000 to $13000 \mathrm{~K}$ ), where the variable ZZ Ceti objects are found (Hébrard et al. 2002b). This line satellite has never been observed in stars or laboratory plasmas. Future FUSE observations and ongoing study of the FUV spectrum of dense hydrogen plasmas may allow this feature to be detected.

Acknowledgements. This work is based on data obtained by the NASA-CNES-CSA FUSE mission operated by the Johns Hopkins University. Financial support to U. S. participants has been provided by NASA contract NAS5-32985. French participants are supported by CNES. The molecular potential computation code was kindly provided by J. Peek; we would like to thank him.

\section{References}

Allard, N. F., Koester, D., Feautrier, N., \& Spielfiedel, A. 1994, A\&A, 108,417

Allard, N. F., Kielkopf, J. F., \& Feautrier, N. 1998a, A\&A, 330, 782

Allard, N. F., Drira, I., Gerbaldi, M., Kielkopf, J. F., \& Spielfiedel, A. 1998b, A\&A, 335, 1124

Allard, N. F., Royer, A., Kielkopf, J. F., \& Feautrier, N. 1999, Phys. Rev. A, 60, 1021

Allard, N. F., Kielkopf, J. F., Drira, I., \& Schmelcher, P. 2000, Eur. Phys. J. D, 12, 263

Allard, N. F., Kielkopf, J. F., Hébrard, G., \& Peek, J. M. 2003, Eur. Phys. J. D, submitted

Baranger, M. 1958, Phys. Rev., 111, 481

Bergeron, P., Wesemael, F., Lamontagne, R., et al. 1995, ApJ, 449, 258

Bragaglia, A., Renzini, A., \& Bergeron, P. 1995, ApJ, 443, 735

Dupuis, J., Vennes, S., Lanz, T., \& Chayer, P. 2001, Am. Astron. Soc. Meet., 199

Dupuis, J., Chayer, P., Vennes, S., Allard, N. F., \& Hébrard, G. 2003, ApJ, submitted

Finley, D. S., Koester, D., \& Basri, G. 1997, ApJ, 488, 375

Greenstein, J. L. 1980, ApJ, 241, L89

Hébrard, G., Lemoine, M., Vidal-Madjar, A. et al. 2002a, ApJS, 140, 103

Hébrard, G., Allard, N., Hubeny, I, et al. 2002b, A\&A, 394, 647

Holberg, J. B., Barstow, M. A., \& Green, E. M. 1997, ApJ, 474, L127

Holberg, J. B., Barstow, M. A., Bruhweiler, F. C., Cruise, A. M., \& Penny, A. J. 1998, ApJ, 497, 935

Holberg, J. B. 2002, 13th European Workshop on WD, Napoli

Holm, A. V., Panek, R. J., Schiffer, F. H., et al. 1985, ApJ, 289, 774

Hubeny, I. 1988, Comp. Phys. Comm., 52, 103

Hubeny, I., \& Lanz, T. 1992, A\&A, 262, 501

Hubeny, I., \& Lanz, T. 1995, ApJ, 439, 875

Kawka, A., et al., 2000, AJ, 120, 3250

Kawka, A., Vennes, S., Koch, R., \& Williams, A. 2002, AJ, 124, 2853

Kielkopf, J. F., \& Allard, N. F. 1995, ApJ, 450, L75

Koester, D., Weidemann, V., Zeidler, K.T., E. M., \& Vauclair, G. 1985, A\&A, 142, L5

Koester, D., \& Allard, N. F. 1993, in White Dwarfs: Advances in Observation and Theory, ed. M. Barstow (Dordrecht: Kluwer), 237

Koester, D., Allard, N. F., \& Vauclair, G. 1994, A\&A, 291, L9

Koester, D., Finley, D. S., Allard, N. F., Kruk, J. W., \& Kimble, R. A. 1996, ApJ, 463, L93

Koester, D., Sperhake, U., Allard, N. F., Finley, D. S., \& Jordan, S. 1998, A\&A, 336, 276

Madsen, M. M., \& Peek, J. M. 1971, Atomic Data, 2, 171

Moos, H. W., et al. 2000, ApJ, 538, L1

Nelan, E. P., \& Wegner, G. 1985, ApJ, 289, L31

Vauclair, G., Schmidt, H., Koester, D., \& Allard, N. 1997, A\&A, 325, 1055

Wolff, B., Kruk, J. W., Koester, D., et al. 2001, A\&A, 373, 674

Wood, B. E., Linsky, J. L., Hébrard, G., et al. 2002, ApJS, 140, 91 